\title{
APLICAÇÃO DA ETAPA P DO CICLO PDCA NO SETOR DE COMPRAS DE UMA DISTRIBUIDORA DE MEDICAMENTOS
}

\author{
Ana Caroline Vitor Barboza - Faculdade da Amazônia (carolinebarboza_19@ hotmail.com) \\ Maitê do Carmo Rocha - Faculdade da Amazônia (mairoccha@gmail.com) \\ Marilia Matos Monteiro Gonçalves Ferreira - Faculdade da Amazônia \\ (mariliazinha@hotmail.com)
}

\section{Resumo}

Mesmo com toda a instabilidade que o mercado brasileiro vem passando, a indústria farmacêutica ainda continua em constante ascensão na economia, mantendo um alto faturamento. Esse fato está diretamente ligado ao aumento da preocupação com a expectativa de vida que se dá através de investimentos e da competitividade das indústrias, que busca de novas tecnologias, lançamentos, aumento da linha de medicamento, entre outros. Esta pesquisa apresenta uma análise no setor de compras em uma distribuidora de alimentos. São direcionadas as formas de compras e controle de estoque para obter a conquista e satisfação do cliente. Este estudo de caso foi desenvolvido em uma distribuidora de medicamentos situada em Ananindeua/PA que atende à região Norte e Sul do Pará. Para a realização deste estudo de caso utilizou-se a coleta de dados e aplicou-se a técnica de brainstorming entre os funcionários da empresa e as pesquisadoras para levantar possíveis causas do problema. $\mathrm{O}$ $5 \mathrm{~W} 2 \mathrm{H}$, uma das ferramentas indispensáveis para o estudo, que seguiu os tópicos propostos na etapa "P" do ciclo PDCA, o planejamento, que consiste em estabelecer métodos para alcançar as metas propostas. Como resultados, concluiu-se que para se ter um bom gerenciamento é necessário mensurar os dados e definir parâmetros para coloca-los em pratica.

Palavras-chave: PDCA, 5W2H, Brainstorming, Planejamento, Distribuidora de medicamentos.

\section{Introdução}

A gestão de compras é uma das atividades mais importantes da área de suprimentos. Através de uma boa gestão é que são selecionados os melhores fornecedores para o 
atendimento das necessidades da organização. "Contudo, a gestão de compras não se limita ao ato de comprar e monitorar. É um processo estratégico, que envolve custo, qualidade e velocidade de resposta. É uma tarefa crucial para a organização, seja de que tipo for" (BERTAGLIA, 2003, p. 27).

A relação entre fornecedores e empresa deve ser cada vez mais próxima, pois o estabelecimento de uma parceria de longo prazo possibilita ganhos para os envolvidos no relacionamento. Para se construir essa parceria é necessário, acima de tudo, confiança, respeito e transparência nas negociações (SEBRAE, 2003).

Conforme Gaither \& Frazier (2001), o departamento de compras desempenha um papel fundamental na realização dos objetivos da empresa. "Sua missão é perceber as necessidades competitivas dos produtos e serviços, tornando-se responsável pela entrega no tempo certo, custos, qualidade e outros elementos na estratégia de operações”.

Nesse sentido, este artigo tem como objetivo analisar o processo de compra de medicamentos. Para atender ao objetivo, foi aplicada a etapa P do ciclo PDCA focado em diagnosticar falhas e erros nos processos, e propor métodos de melhorias através das ferramentas da qualidade.

Neste contexto, a pesquisa possui como problemática o seguinte questionamento: quais os principais motivos para o erro nas compras na empresa de distribuição de medicamentos?

Com intuito responder tal problemática, este artigo propôs uma abordagem exploratória e descritiva por meio de um estudo de caso em uma empresa. O tema da pesquisa possui grande importância para o estudo na área de engenharia de produção, pois é focado no diagnóstico de falhas no desenvolvimento das atividades realizadas, e mostra o favorecimento da aplicação das ferramentas da qualidade nesta empresa.

\section{Controle de estoque}

O controle de estoque deve ser utilizado tanto para matéria prima, mercadorias produzidas e/ou mercadorias vendidas. Para conseguir um bom controle de estoque, o primeiro passo seria ter um bom e confiável sistema que lhe auxilie na administração e organização de todo o material de forma que ele consiga ainda realizar suas outras funções.

É praticamente impossível prever com exatidão a demanda futura, tornando-se necessário manter determinado nível de estoque, para assegurar disponibilidade de produtos 
(POZO, 2010). Entretanto, estoques absorvem capital que poderia ser aplicado em outros investimentos. Elevar a rotatividade, libera ativo e economiza o custo de manutenção do estoque. Dessa forma, é preciso uma política de estoque adequada, para que não se tenha material em excesso e nem em falta (NOGUEIRA, 2012).

\section{Compras}

O setor de compras é hoje o mais importante dentro de uma empresa, pois ele tem sido estudado e atualizado através de novas estratégias para que com eficiência ele consiga reduzir os custos, trazer novidades, abranger a quantidade de clientes e ofertar novos serviços.

Segundo Dias e Costa (2012), a área de compras tem como objetivo: "adquirir bens e/ou serviços, na qualidade desejada, no momento preciso, pelo menor custo possível e na qualidade pedida." Mais detalhadamente esses autores abordam cada um dos tópicos:

Hoje o departamento em questão não trabalha, mas isoladamente como antes, onde fazia apenas a função de compra, atualmente ele trabalha de forma integrada com todos os outros setores da empresa, pois, dessa forma consegue acompanhar o pedido do cliente, quais as marcas mais usadas, valores, qualidade e principalmente dentro do prazo do acordo.

Saber comprar de forma a beneficiar o empreendimento é determinante não somente para a competividade, mas também para a permanência da empresa no mercado.

Compras exige todo um planejamento para poder diminuir os custos, e dentro deste se destaca os quatros pontos cruciais

a) A quantidade solicitada pelo cliente deve ser cumprida, pois muitas das vezes o cliente segue um padrão. Ex: um hospital fecha um medicamento como anestesia com o distribuidor da marca ABL e recebe o medicamento da marca TEUTO onde o setor de anestesia já tem o padrão daquela anestesia testado e não pode ser trocado, pois, a eficácia de outro produto implicará no resultado dos procedimentos ofertados pelo hospital e posteriormente esse erro de compras pode refletir na qualidade de serviço do mesmo.

b) No momento preciso: essa etapa consiste na agilidade da entrega acordada, caso contrário nem seja, mas necessário a compra do produto. Ex: um hospital realizará uma ação de cirurgia em uma data especifica e precisará de um determinado medicamento, mas ocorre o atraso da entrega e esse material chega um dia depois da ação. 
c) Menor custo está ligado a quantidade e o prazo de entrega onde será verificado qual o menor custo de transporte, mas que atenda aos requisitos anteriores.

d) A quantidade pedida deve ser entregue de forma integral para que assim a compra seja finalizada com sucesso, caso contrário o processo de compra ainda fica em aberto e podendo correr risco de não ter previsão para a entrega da outra parte dos itens.

Já para Baily (2000) as atividades são pertinentes à função de compras:

a) Assegurar continuidade de suprimentos para manter relacionamentos efetivos com fontes existentes, desenvolvendo outras fontes de suprimentos alternativas, ou para atender as necessidades emergentes ou planejadas;

b) Comprar eficiente e sabidamente, obtendo por meios éticos o melhor valor por centavo gasto;

c) Administrar estoques para proporcionar o melhor serviço possível aos usuários e ao menor custo;

d) Desenvolver funcionários, políticas, procedimentos e organização para assegurar o alcance dos objetivos previstos;

e) Selecionar os melhores fornecedores do mercado;

f) Ajudar a gerar o desenvolvimento eficaz de novos produtos;

g) Proteger a estrutura de custos da empresa;

h) Manter o equilíbrio correto de qualidade/valor;

i) Negociar eficazmente para trabalhar com fornecedores que buscarão benefício mútuo.

A aplicação de compras, requer escolha de materiais, fornecedores, armazenamento, entrega. Sendo assim compras é um elemento indispensável na cadeia de suprimentos. Segundo Zsidisin; Siferd (2001) as compras atuam na fronteira que conecta a firma com os fornecedores que lhe provêm os bens e serviços necessários para a produção de serviços da empresa compradora.

\section{Método PDCA e ferramentas da qualidade}

O método PDCA (Plan - Planejar; Do - Executar, Check - Controlar, Action - Auar) é uma ferramenta indispensável no ambiente organizacional, tem seu método gerencial para melhoria de processos e soluções de problemas, sendo a base da melhoria contínua, podendo ser utilizado em qualquer tipo de organização, seja em uma microempresa, uma organização sem fins lucrativos ou até mesmo em um setor público. 
Campos (1992), diz que o controle de processos deve ser executado de acordo com o método PDCA, demonstrado na figura 1, para atingir as metas necessárias para sobrevivência da empresa.

Figura 1 - PDCA - Método de Controle de Processos

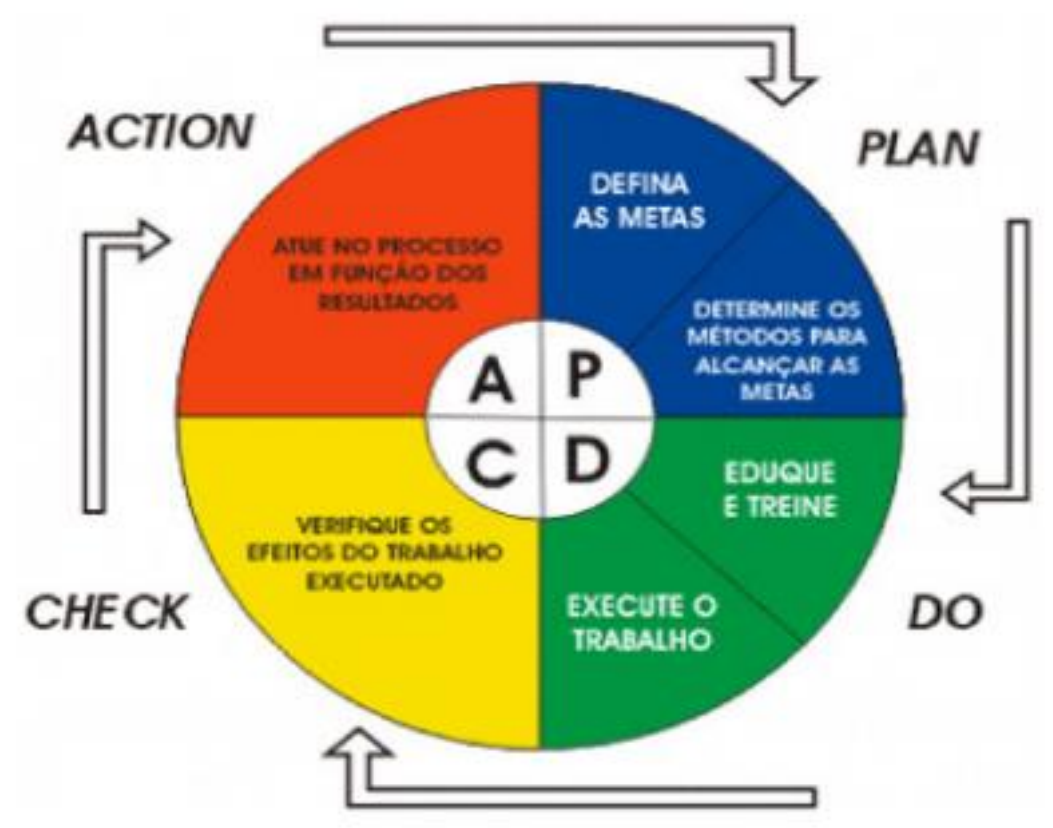

Fonte: Campos, 1996, p.266.

Segundo Paladini (2012), as ferramentas da gestão da qualidade como mecanismos simples para selecionar, implantar ou avaliar modificações no processo produtivo por meio de análises objetivas de partes bem definidas deste processo. Em outras palavras, o objetivo das modificações é gerar melhorias. Colocando-as em pratica de forma cíclica e sem interrupção, acaba-se por favorecer a melhoria contínua e sistemática na organização, estabelecendo a padronização de práticas. Algumas destas ferramentas serão descritas a seguir.

\subsection{Diagrama de Ishikawa e Brainstorming}

O diagrama de Ishikawa simplifica processos considerados complexos dividindo-os em processos mais simples e, portanto, mais controláveis (TUBINO, 2000). Esta ferramenta é um método bastante efetivo na busca das raízes do problema (SLACK, 2009).

De acordo com Moura (2003), está é uma ferramenta útil para análise dos processos de forma a identificar as possíveis causas de um problema. De acordo com Campos (1999), são elas: máquinas, meio ambiente, medidas, materiais, métodos e mão-de-obra, conforme ilustrado no diagrama da figura 2 . 
Figura 2 - Diagrama de Ishikawa e ilustração dos 6M

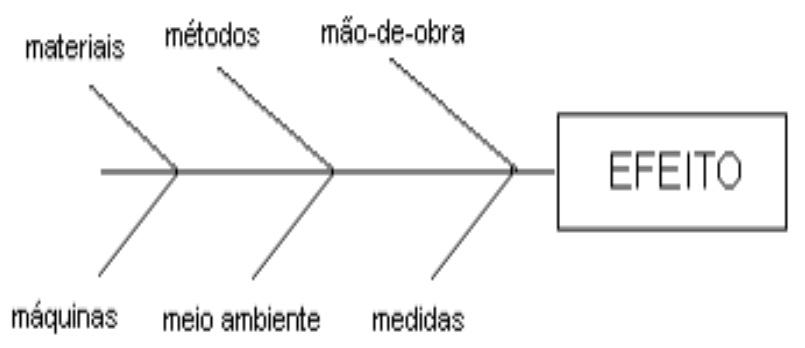

Fonte: Campos, 1999.

Brainstorming é uma técnica de geração de ideias. Na língua inglesa, o termo brain significa cérebro enquanto que storming significa tempestade. A versão, na língua portuguesa, seria uma "explosão de ideias" (MINICUCCI, 2001). É uma maneira de geração de novas ideias a partir de discussão em grupo, onde os mesmos podem expressar suas ideias e opiniões.

Diante do assunto fica evidenciada a importância da utilização, tanto da construção do diagrama de Ishikawa para apresentação das causas de problemas, assim como a realização do brainstorming, na busca pela identificação destas causas.

\section{$4.25 \mathrm{~W} 2 \mathrm{H}$}

A ferramenta $5 \mathrm{~W} 2 \mathrm{H}$ foi introduzida por profissionais do ramo automobilístico do Japão com intuito de auxiliar a utilização da ferramenta de qualidade PDCA, principalmente em seu planejamento (SILVA et al., 2013).

No planejamento de um plano de ação elabora-se uma estratégia, promovendo reuniões com um grupo de pessoas envolvidas a fim de definir um plano com base na estrutura 5W2H (WERKEMA, 1995). Um bom plano de ação deve deixar claro tudo o que deverá ser realizado e, assim, desenvolver um pequeno checklist, apontando os principais pontos de ação (MESQUITA e VASCONCELLOS, 2009).

Esta ferramenta deve descrever um plano de ação com as atividades que precisam ser desenvolvidas com a maior clareza possível para obter o entendimento do funcionário que irá executá-la. Suas aplicações, podem ser observadas na Quadro 1: 


\begin{tabular}{|c|c|c|c|}
\hline \multicolumn{4}{|c|}{ Método dos 5W 2H } \\
\hline \multirow{5}{*}{$5 \mathrm{~W}$} & What & O que? & Que ação será executada? \\
\hline & Who & Quem? & Quem irá ex ecutar/participar da ação? \\
\hline & Where & Onde? & Onde será ex ecutada a ação? \\
\hline & When & Quando? & Quando a ação será ex ecutada? \\
\hline & Why & Por quê?? & Por que a ação será ex ecutada? \\
\hline \multirow{2}{*}{$2 \mathrm{H}$} & How & Como? & Como será ex ecutada a ação? \\
\hline & How much & Quanto custa? & Quanto custa para executar a ação? \\
\hline
\end{tabular}

Fonte: Adaptado de Meira (2003)

\section{Metodologia}

A pesquisa foi realizada através de questionários, coleta de dados e aplicação da técnica de brainstorming entre os funcionários da empresa e as pesquisadoras para levantar possíveis causas do problema, com intuito de conhecer um pouco mais da rotina de cada setor, para entender através da observação e analisar o grau de integração entre os setores e verificar como a forma que as atividades são executadas tendem a inferir na qualidade do setor de compras.

A empresa em questão, está no mercado há 18 anos e tem em cerca de 35 funcionários divididos nos setores de licitação, comercial, logística, financeiro e administração. Busca atender a todas as cidades do estado do Pará com agilidade e qualidade.

Percebe-se no decorrer da pesquisa, que o setor de compras não pode trabalhar sozinho, pois, ele é o centro da empresa e precisa estar interligado a todo e qualquer decisão para que assim consiga contribuir de forma correta e eficaz com a gestão da empresa e os resultados financeiros.

\section{Análise dos resultados}

No primeiro momento, foi realizado um acompanhamento no setor de televendas durante 1 semana para verificar qual o nível atendimento entre cliente e o departamento, pois através dessa análise foi notório que a relação entre cliente-vendedor era bem alinhada com apenas uma classe que corresponde a $20 \%$ em relação a todos os clientes cadastrados. Esse levantamento foi necessário para entender que dentro do atendimento desses $20 \%$ poucos vendedores tem o domínio e acompanhamento necessário para ditar a demanda e quais os 
itens e marcas que seus clientes usam, e o seu período de compra sazonal. Essas informações são de extrema importância para ressaltar o que deve ser comprado e o que não tem saída. Feito isso, verificou-se que o sistema utilizado na empresa não possuía um espaço para depositar essas informações que pudessem programar e gerar relatórios mais assertivos para auxiliar nas compras.

Foi aplicado posteriormente a ferramenta de Ishikawa para auxiliar no brainstorming a verificação de outros fatores que influenciam no setor de compras.

Figura 3 - Ferramenta de Ishikawa para auxilio no brainstorming

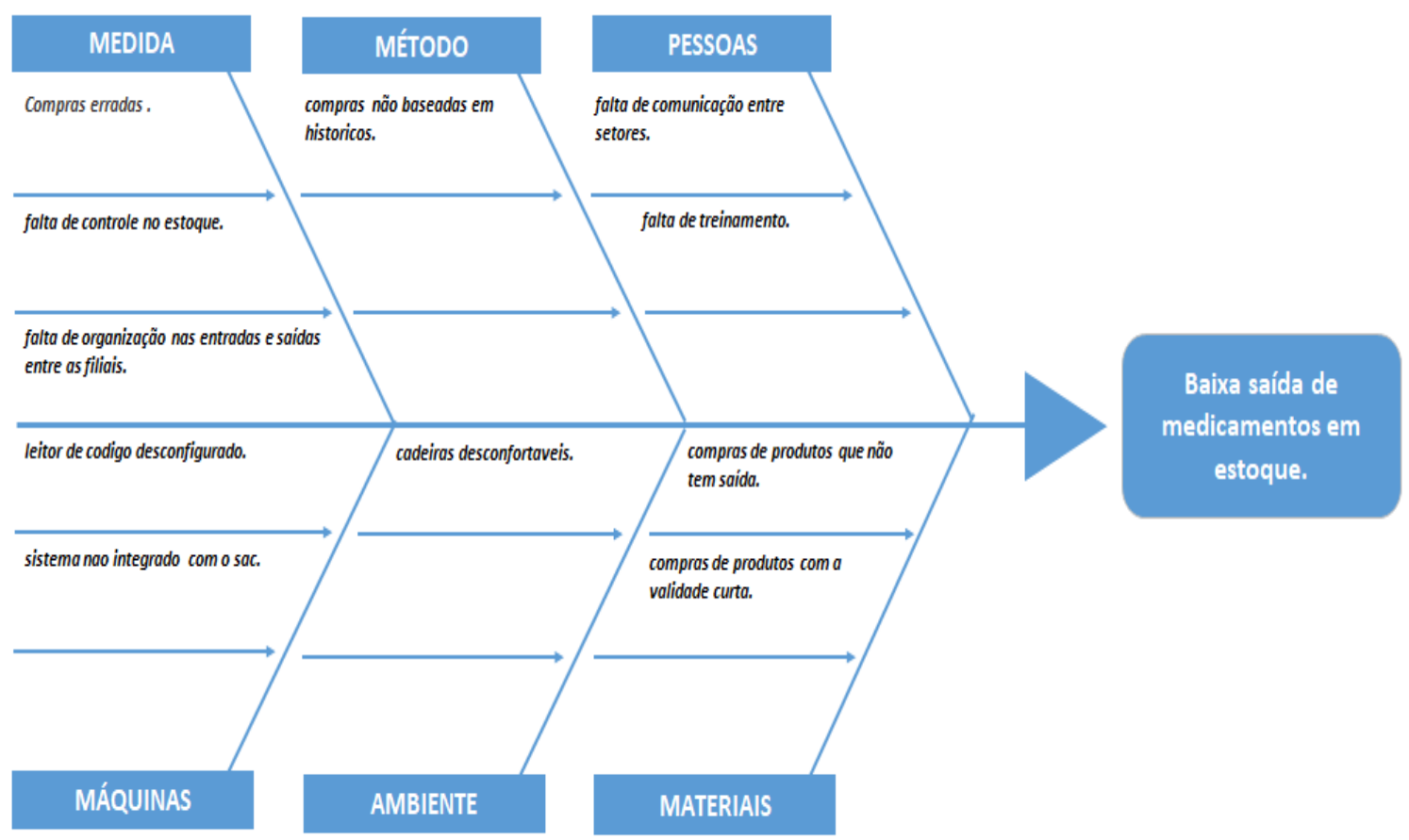

Fonte: Elaborado pelos Autores.

Através da aplicação desta ferramenta foi possível perceber e descrever os gaps principais.

No tópico "pessoas" foi perceptível a falta de treinamento dos funcionários, pois as atividades exercidas pelos mesmos eram realizadas com o mínimo de informações possíveis dificultado assim uma eficácia no trabalho de atendimento, no setor de compras e setor da logística. No atendimento as informações não transmitiam firmeza e segurança do produto vendido ou falta de explicação correta de que um medicamento tem vários nomes dependendo do laboratório, mas que a sua base sempre será a mesma. No setor de compras houve uma troca recente da pessoa responsável onde a última compra foi realizada sem base de dados ou 
histórico dos itens que saem mais, outro fator que deveria ser realizado, é o diálogo entre vendedor e comprador, deve ser passado a lista individual da demanda de medicamentos por vendedor para que junto com o setor de compras possa ser realizado um tipo de compra programada, mas na realidade não é passado nenhum relatório que contenha essas informações. Na logística geralmente acontece um erro na hora da conferencia, ou na hora da entrega trocar os materiais de uma clínica com um hospital por exemplo.

O método de compras é realizado de forma ineficiente, pois uma vez que não se tem uma pesquisa de mercado para saber quais os clientes que serão atendidos e suas demandas, o fato de comprar mal ou não saber comprar pode gerar um grande problema para a saúde financeira da empresa. Dentro do tópico chamado de medidas trata sobre a compra de forma errônea que impacta diretamente no capital parado, pois uma vez que o produto não tenha giro no mercado ele se torna um tipo de perda. Outro gap que chamou atenção dentro da empresa, foi na logística, ao ser realizada uma análise no sistema e o dia a dia de vendas, foi possível notar que o sistema não estava de acordo com o estoque físico, esse fato pode implicou na perda de fidelidade de um cliente, onde o mesmo liga para a empresa, faz a cotação, confirma o pedido e na hora do faturamento somente na hora da separação é verificado o erro de que o produto consta no sistema e não consta no estoque físico. Podendo gerar problemas para o cliente gerando o retrabalho e também gastos para a empresa devido o cancelamento de NF. Dentro do tópico chamado de medidas foi possível perceber a faltar de organização para controlar o movimento de entradas e saídas entre as filiais, e dessa forma levando a custo mais altos pois alguns materiais simplesmente não eram encontrados no meio dessa transição.

Ao verificar-se o processo na etapa máquinas o que chamou mais atenção foi a falta de eficiência do leitor de código pois muitas das vezes durante o bip o leitor dava erro e de 5 funcionários da logística apenas 2 sabiam usar de forma correta. Sem esse controle alinhado do estoque físico e virtual é impossível organizar e ajudar o setor de compras, assim como o sistema utilizado na distribuidora que não oferece alguns relatórios mais precisos, para se ter essas informações é preciso extrair os dados de algumas janelas e passar para o Excel e assim ir colhendo os dados necessários, o que leva tempo, dedicação e atrapalha na produtividade do funcionário.

Quando o ambiente foi analisado o único fator que implicava ergonomicamente era a cadeira que não se ajustava a altura da mesa para alguns funcionários com mais de 1,80m, inclusive no setor de compras, esse fator implica na produtividade e bem-estar de cada um, podendo diminuir a eficácia das atividades e gerar danos ao funcionário. 
O último ponto explanado foram os materiais. Acompanhamos quais os indicadores de compras para entender se existem algo que possa ser mudado no resultado de falta de vendas de mercadorias e compras erradas. Ao acompanharmos o setor, pode-se perceber que eram realizadas compras de alguns medicamentos com validades com menos de um ano para que assim o comprador adquira um tipo de desconto, que acaba não se tornando viável levando em consideração o volume com o fluxo de saída do produto e a vida útil , esse fator está diretamente ligado na saída do produto, umas vez que a maioria de seus clientes durante o tramite de negociação destaca que o material ou medicamento a ser negociado deve estar com no mínimo a

1 ano de validade. Além da validade curta as compras são realizadas de forma aleatória de modo que para fechar uma variedade de medicamentos e materiais com a indústria farmacêutica é posto $50 \%$ de materiais que não tem giro, e acaba ficando parado ao invés de aumentar o volume em produtos que são considerados o carro feche da empresa.

Após ser realizados a aplicação do brainstorming foi aplicado também o 5W2H afim de fazer um planejamento para aplicação de ação e melhorias de forma simples e objetiva estabelecendo o tempo em que a ação deve começar e terminar.

Quadro 2 - Aplicação do 5W2H

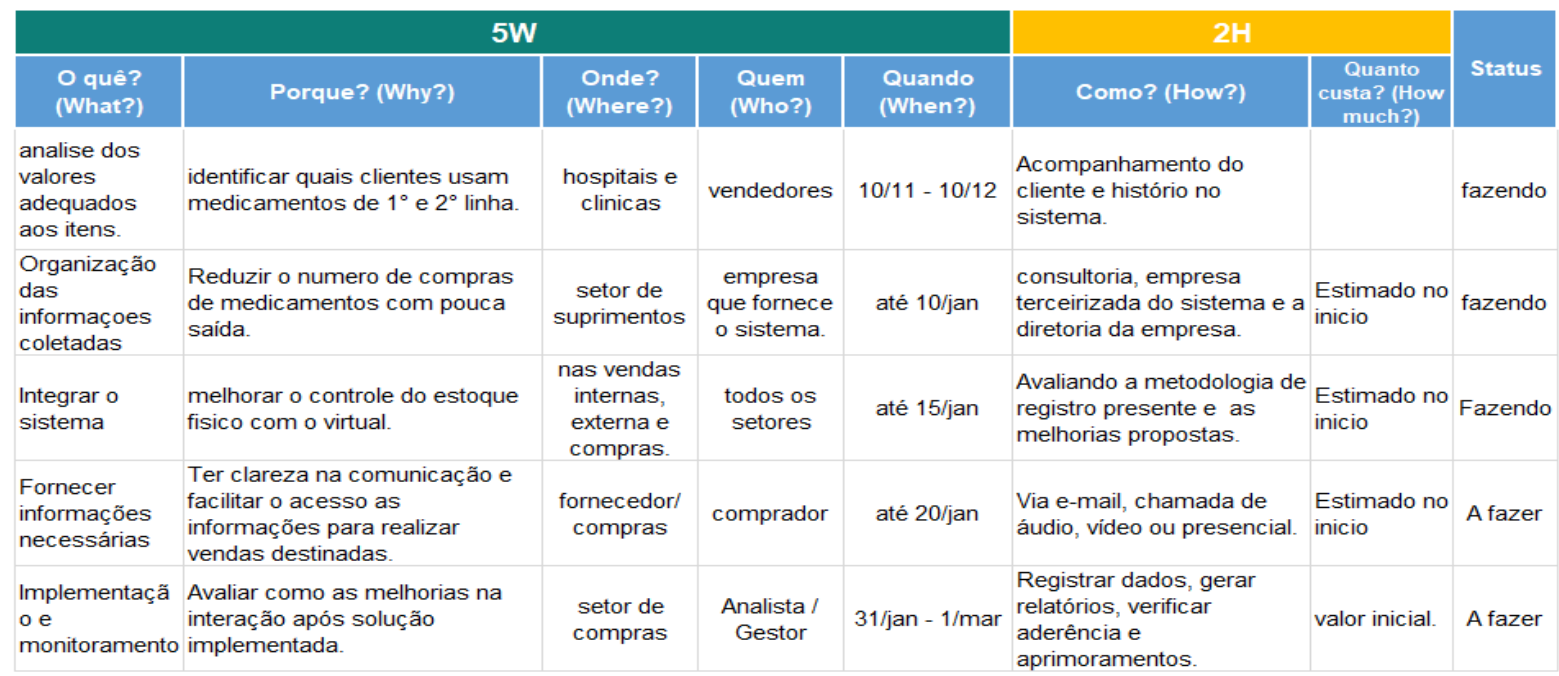

Fonte: Elaborado pelos Autores.

Posteriormente após algumas solicitações de melhorias junto a diretoria, alguns dos planos de ação já deram início, todas voltadas com mais dedicação e melhor gestão dentro do setor de compras. A empresa que presta serviço de SAC também passou uma semana 
acompanhando o setor de compras para entender qual a necessidade de melhoria na plataforma.

Contudo através de analises foi possível chegar à conclusão de que, para se ter um bom gerenciamento é necessário mensurar os dados e definir parâmetros para coloca-los em pratica.

No último levantamento realizado através de planilhas foi possível visualizar a marca que possui mais saída na distribuidora e a partir desta aumentar as compras nos itens de forma assertiva como mostra o gráfico a seguir com a marca ABL levando 50\% de faturamento num período trimestral de novembro de 2019 a janeiro de 2020 .

Gráfico 1 - Faturamento num período trimestral de novembro de 2019 a janeiro de 2020.

\section{PORCENTAGEM DE VENDAS ENTRE AS MARCAS QUE A DISTRIBUIDORA TRABALHA}

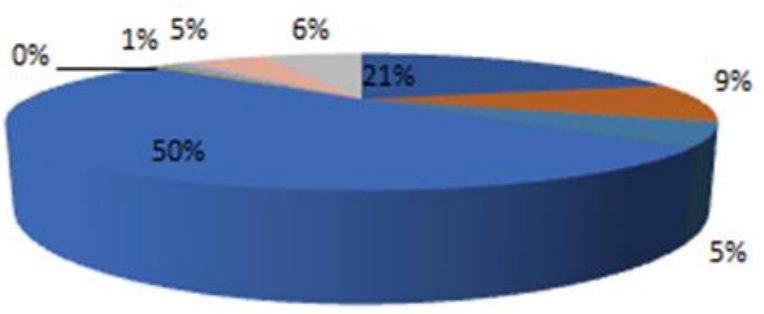

Fonte: Elaborado pelos Autores.

Observa-se na tabela 2 que as marcas ficaram distribuídas com porcentagens referentes as saídas:

Tabela 1 - Distribuição das porcentagens

\begin{tabular}{|c|c|c|c|}
\hline Rótulos de & MARCA & Total & $\%$ \\
Linha & Geral & \\
\hline
\end{tabular}




\begin{tabular}{|c|c|c|c|}
\hline 002022 & BD & 28738,1 & $21 \%$ \\
\hline 002078 & CRISTALIA & 12365,12 & $9 \%$ \\
\hline 002115 & PRATI & 186 & $0 \%$ \\
\hline 002147 & RIOQUIMICA & 148,32 & $0 \%$ \\
\hline 002216 & MUCAMBO & 6927,3 & $5 \%$ \\
\hline 002555 & SOBRAL & 208 & $0 \%$ \\
\hline 002634 & ABL & 68815 & $50 \%$ \\
\hline 002698 & REALI M. & 150 & $0 \%$ \\
\hline 002724 & PHARLAB & 627 & $0 \%$ \\
\hline 002918 & CPL & 315,5 & $0 \%$ \\
\hline 003023 & BIOSANI & 344 & $0 \%$ \\
\hline 003355 & MEDLEVN. & 260 & $0 \%$ \\
\hline 003929 & ABDESC & 1575 & $1 \%$ \\
\hline 003951 & BEKER & 7138 & $5 \%$ \\
\hline 004189 & ROBISA & 8480 & $6 \%$ \\
\hline TOTAL LIQ.: & & 136277,3 & $100 \%$ \\
\hline
\end{tabular}

Fonte: Elaborado pelos Autores.

\section{Conclusão}

Com o intuito de acrescentar conhecimento à empresa, melhorar os seus processos e aumentar o seu lucro, a pesquisa buscou recomendar procedimentos a fim de eliminar esses problemas.

Identificado que o setor de compras pode afetar o rendimento na venda de medicamentos, foi possível visualizar a marca que possuía mais saída na distribuidora e a partir desta aumentar as compras nos itens de forma segura

O trabalho realizou um diagnóstico do setor de compras e identificou problemas relacionados ao fluxo organizacional, na elaboração das cotações e não esquecendo de ressaltar a identificação da insegurança dos funcionários em transmitirem com firmeza e segurança o produto ou falta de explicação correta de que um medicamento possui outros nomes, dependendo do laboratório. Com isso, foi identificado que essa falta de padronização nos métodos de compra ocasiona na distribuição com qualidade dos medicamentos e consequentemente afeta a venda. 
Após a análise de todos os problemas identificados sugere-se a empresa que reavalie a estrutura organizacional do processo de compras, pois com a aplicação da ferramenta que compõe a etapa $\mathrm{P}$ foi possível perceber que mesmo com essas ferramentas mais simples é possível visualizar como está a gestão da empresa, descobrir as suas causas, e criar planos de ação para uma forma de organização mais eficaz que no caso foram propostos reajustes no sistema da empresa para gerar as informações com precisão para auxiliar no setor de compras e através dessa metodologia uma vez que as compras aumente a qualidade no serviço os outros setores interligados também serão realizados com mais qualidade.

\section{Referências}

BERTAGLIA, P. R. Logística e gerenciamento da cadeia de abastecimento. São Paulo: Saraiva, 2003.

CAMPOS, V. F. Gerenciamento da rotina do trabalho do dia-a-dia. Minas Gerais; INDG Tecnologia e Serviços Ltda., 1999.

CAMPOS, Vicente Falconi. Controle da Qualidade Total. Rio de Janeiro: Editora Bloch, $3^{\text {a }}$ edição, 1992.

DIAS, Mario; COSTA, Roberto Figueiredo. Manual do comprador. São Paulo: Saraiva, 2012.

GAITHER, Norman; FRAZIER, Greg, Administração da Produção e Operações. 8 ed. São Paulo: Pioneira, 2001.

MEIRA, R. C. As ferramentas para a melhoria da qualidade. 2. Ed. Porto Alegre: SEBRAE/RS, 2003.

MINICUCCI, A. Técnicas do trabalho de grupo. São Paulo: Atlas, 2001.

MOURA, L. R. Qualidade Simplesmente Total. Rio de Janeiro: Qualitymark, 2003. 
NOGUEIRA, A. Logística Empresarial: Uma visão local com pensamento globalizado. 1 ed. São Paulo: Atlas, 2012

DIAS, Mario; COSTA, Roberto Figueiredo. Manual do comprador. São Paulo: Saraiva, 2012.

PALADINI E. P. Gestão da qualidade: teoria e prática. $3^{\text {a }}$ ed. Rio de Janeiro: Atlas, 2012.

POZO, H. Administração de Recursos Materiais e Patrimoniais. 6. ed. São Paulo: Atlas, 2010 .

SERVIÇO BRASILEIRO DE APOIO ÀS MICRO E PEQUENAS EMPRESAS (SEBRAE). Ganhe mais com menos fornecedores. 2003. Disponível em: Acesso em: maio. 2012.

SILVA, A. O.; RORATTO, L.; SERVAT, M. E.; DORNELES, L.; POLACINSKI, E. Gestão da qualidade: Aplicação da ferramenta 5W2H como plano de ação para projeto de abertura de uma empresa. In: $3^{\text {a }}$ Semana Internacional das Engenharias da FAHOR. Anais... Horizontina, 2013.

SLACK, N.; CHAMBERS, S.; HARLAND, C.; HARRISON, A.; JOHNSTON, R. Administração da Produção; Revisão técnica Henrique Corrêia, Irineu Giaresi. São Paulo: Atlas, 2009.

TUBINO, D. F. Manual de planejamento e controle da produção. São Paulo: Atlas, 2000.

WERKEMA, Maria Cristina Catarino. Ferramentas estatísticas básicas para o gerenciamento de processos. Belo Horizonte: UFMG, Escola de Engenharia, 1995. 